\title{
Role of plaque characterization by 64-slice multi detector computed tomography in prediction of complexity of percutaneous coronary interventions
}

\author{
Sherif Wagdy Ayad*, Mohamed Ahmed Sobhy, Eman Mohamed El-Sharkawy and Mahmoud Shehata Abd-Elhamid \\ Faculty of medicine, Alexandria University, Egypt
}

\begin{abstract}
Background: Percutaneous coronary intervention (PCI) nowadays has become standard of care in symptomatic coronary artery disease. (CAD). Lesion localization, severe calcifications, and vessel tortuosity may challenge the skills of the operator and increase the risk of procedural complications. Coronary computed tomography angiography (CCTA) has become a promising non-invasive imaging technique, preprocedural lesion characterization by CCTA might contribute to predict PCI complexity in extensive and complex CAD.
\end{abstract}

Aim: to determine the predictive value of coronary plaque characterization as detected by 64- slice multi-detector Computed tomography (MDCT) in prediction of the procedural complexity of elective percutaneous coronary non CTO intervention.

Patients: retrospective study involving 45 patients in whom PCIs were performed for 60 single coronary lesions within 6 months of pre-procedural CCTA at ICC hospital.

Methods: All patients had MDCT coronary angiography The CT derived parameters included in our study was: calcium scoring, plaque anatomy (site, side branch involvement, degree of stenosis, morphological type), plaque length, volume, density and area.

Results: Median Agatston score was significantly higher in lesions with complex as compared to those with non-complex interventions with a mean of $266.51 \pm$ 155.93 vs. $168.76 \pm 70.53$ with the $\mathrm{P}$ value $=0.034$. Regarding plaque density, Hounsfield Units were higher in lesions with complex as compared to non-complex PCI (371.85 (48.0 to 1159.0) vs. 232.29 (52.0 to 1307.0) Hounsfield Units; P=.033). Median local plaque volume was higher in lesions with complex PCI compared to those with non-complex interventions with a mean of 475.07 (92.0 to 1229.0$) \mathrm{mm}^{3}$ vs. 270.24 (69.0 to 824.0$)$ mm ${ }^{3}$. The plaque area was found statistically significantly higher in complex PCI procedures over the non-complex ones with the mean plaque area in the complex procedures was $19.40 \pm 14.61$ mm ${ }^{2}$ and in the non-complex ones, it was $11.24 \pm 7.94 \mathrm{~mm}^{2}$ with the $\mathrm{P}$ value $=0.011$. Complex PCI had a higher rate of calcified plaques $(27.9 \%$ vs. $5.9 \%$; $P=.046)$.

Conclusion: Pre-procedural CCTA parameters indicate complexity of PCI. Thus, we suggest that in patients with suspected complex coronary anatomy, prior CCTA adds important complementary information to coronary angiography for planning subsequent PCI strategy.

\section{Introduction}

Percutaneous coronary intervention (PCI) nowadays has become standard of care in symptomatic coronary artery disease (CAD) with suitable anatomy, and it is increasingly used in the treatment of complex CAD [1]. However, overall lesion complexity impacts in many aspects on the procedure itself, as well as on short- and longterm outcome [2,3]. Lesion localization, severe calcifications, and vessel tortuosity may challenge the skills of the operator and increase the risk of procedural complications such as coronary artery dissection, perforation, and plaque shift. Hence, comprehensive pre-procedural patient evaluation and lesion characterization are fundamental. In recent years, coronary computed tomography angiography (CCTA) has become a promising non-invasive imaging technique, mostly applied prior to coronary angiography. Although preferentially used in patients with low to intermediate probability of CAD to avoid invasive diagnostics for exclusion of the condition in these patients, [4] CCTA provides nevertheless important incremental information to coronary angiography with regard to lesion characterization and threedimensional coronary anatomy.
So far, the potential incremental information of CCTA for guiding PCI has been studied only for chronic total occlusions (CTO), although suggestions for a broader use have been made [5-7]. Indeed, lesion length $>15 \mathrm{~mm}$, target vessel tortuosity, and severe calcification assessed by CCTA and unenhanced computed tomography (CT) have been identified as independent predictors of procedural failure for CTO [8-14].

Thus, we hypothesized that preprocedural lesion characterization by CCTA and unenhanced CT might contribute to predict PCI complexity in extensive and complex CAD. To support this hypothesis and to provide the rationale for future research, we analyzed retrospectively the correlation between specific CCTA parameters and indicators of complex PCI, an operational study definition for the purpose of this study, comprising use of buddy wire, kissing balloon,

Correspondence to: Sherif Wagdy Ayad, Faculty of medicine, Alexandria University, Egypt, E-mail: sherifwagdyayad@yahoo.com

Received: July 12, 2015; Accepted: August 04, 2015; Published: August 07, 2015 


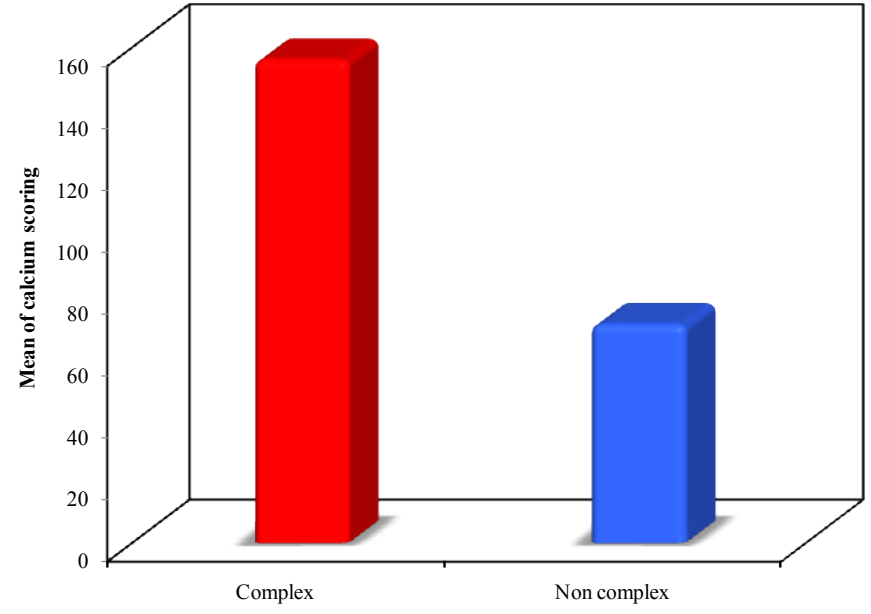

Figure 1. Comparison between studied groups according to calcium scoring.

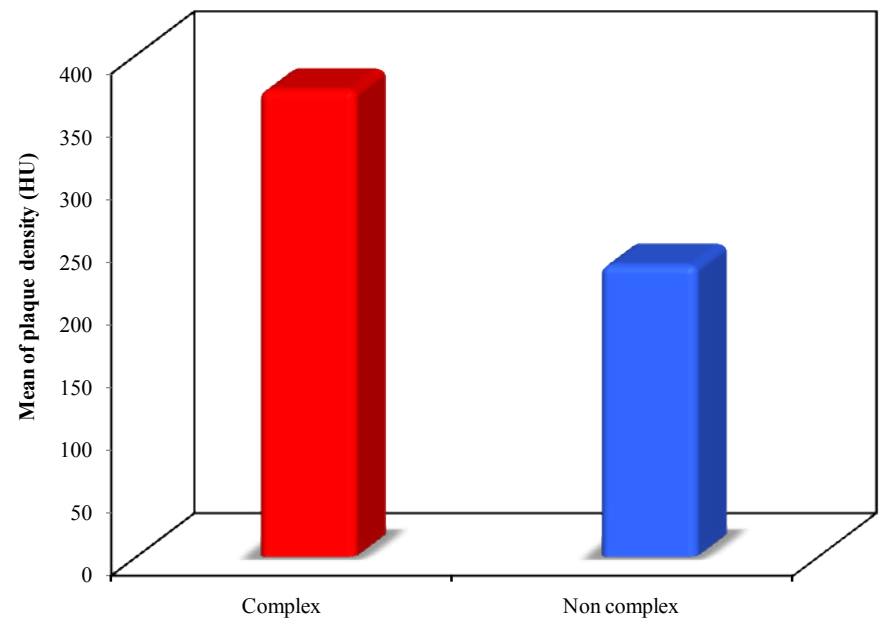

Figure 2. Comparison between studied lesions according to Plaque density (HU).

necessity of high-pressure balloon, or use of rotablator due to heavy calcifications. Pre-procedural lesion characterization by CCTA might provide important additional information on lesion complexity prior to invasive diagnostics, and thus may allow for early stratification of the most appropriate PCI strategy.

\section{Methods}

\section{Patients}

The study population involved 45 patients in whom PCIs were performed for 60 single coronary lesions within 6 months of preprocedural CCTA. All patients were subjected to thorough history taking, clinical examination, renal function testing and 12 lead ECG.

\section{4-Slice MDCT coronary angiography}

Images were acquired using 64-MDCT (Aquilion 64 Toshiba Medical system). Reconstruction was obtained at $75 \%$ of the R-R interval for most of the cases. Reconstruction in more phases was left for the reader's preference. The image analysis was done using Vitrea 3 work station by 3D multi-planar reconstruction, axial images, curved multi-planar reconstruction (MPR), volume rendering and multiple intensity projection (MIP). Two reviewers in consensus evaluated the axial images, three-dimensional (3D) reconstruction, and vessel tracking, for the existence of significant stenosis $(>50 \%$

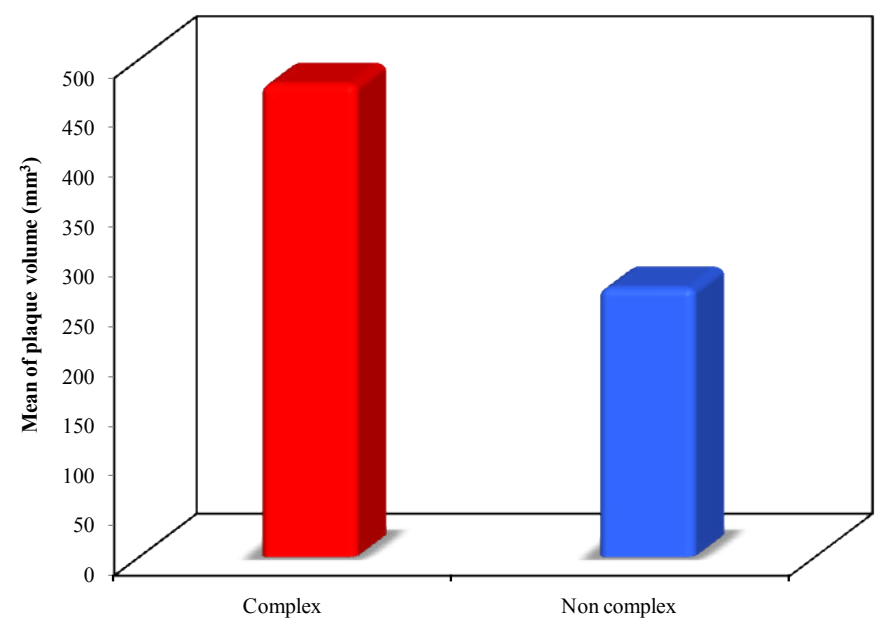

Figure 3. Comparison between studied lesions according to Plaque volume $\left(\mathrm{mm}^{3}\right)$.

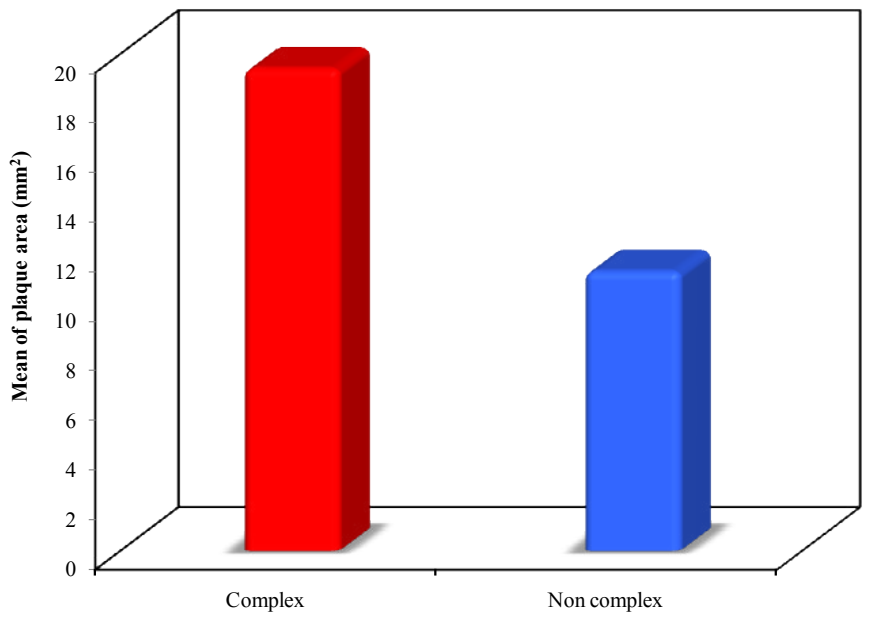

Figure 4. Comparison between studied lesions according to Plaque area $\left(\mathrm{mm}^{2}\right)$.

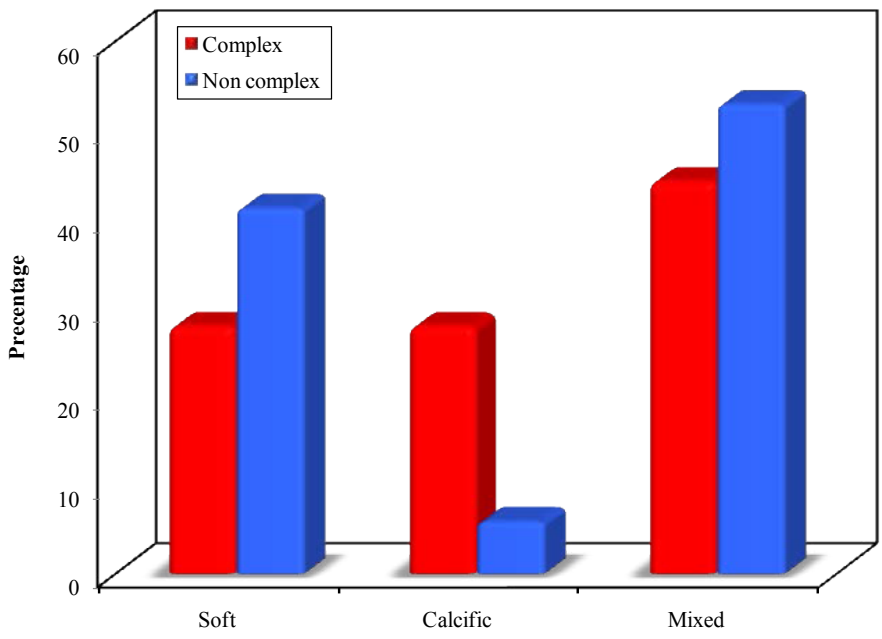

Figure 5. Comparison between studied groups according to Plaque type. 


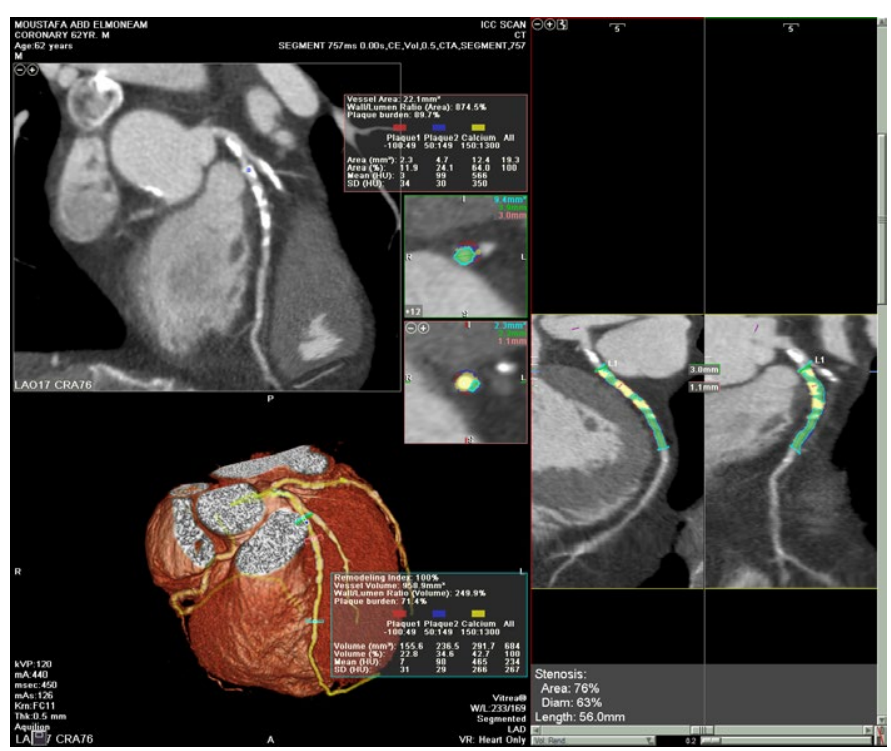

Figure 6. Curved multiplanar reconstruction (MPR) of the LAD showing a long mid LAD plaque. The bottom left image represent a volume rendered image showing diffusely atherosclerotic $\mathrm{LAD}$.

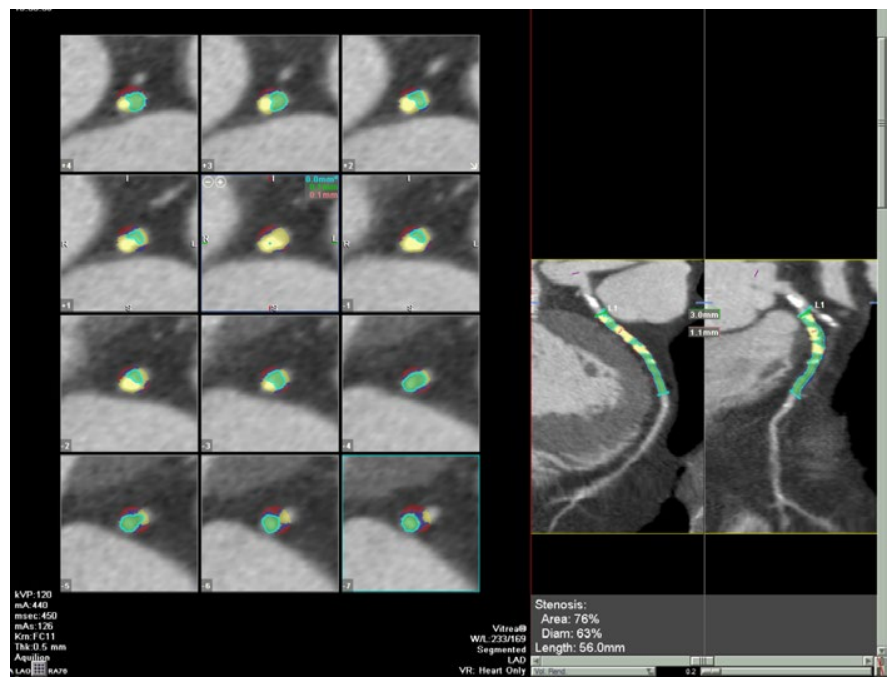

Figure 7. MPR image of LAD revealing the site of maximum stenosis.

lumen reduction). The CT derived parameters included in our study was: calcium scoring, plaque anatomy (site, side branch involvement, degree of stenosis, morphological type), plaque length, volume, density and area.

\section{Analysis of coronary angiography and PCI}

Complex PCI was defined as use of buddy wire, kissing balloon, and necessity of high-pressure balloon pre- or post-dilatation, use of anchor technique, mother in child technique, or use of rotablator due to calcifications [15]. High pressure balloon applied in calcified lesions and in cases of angiographically or IVUS-documented under expansion reflects complex anatomy.

Complications were recorded according to the American Heart Association \&American College of Cardiology guidelines for PCI [16]. Procedure related complications are following: dissection, perforation, stent dislodgement, under-deployment, plaque shift.

\section{Statistical analysis}

Continuous variables are presented as medians and interquartile ranges. Categorical variables are given as frequencies and percentages. Testing of significance was done using appropriate tests.

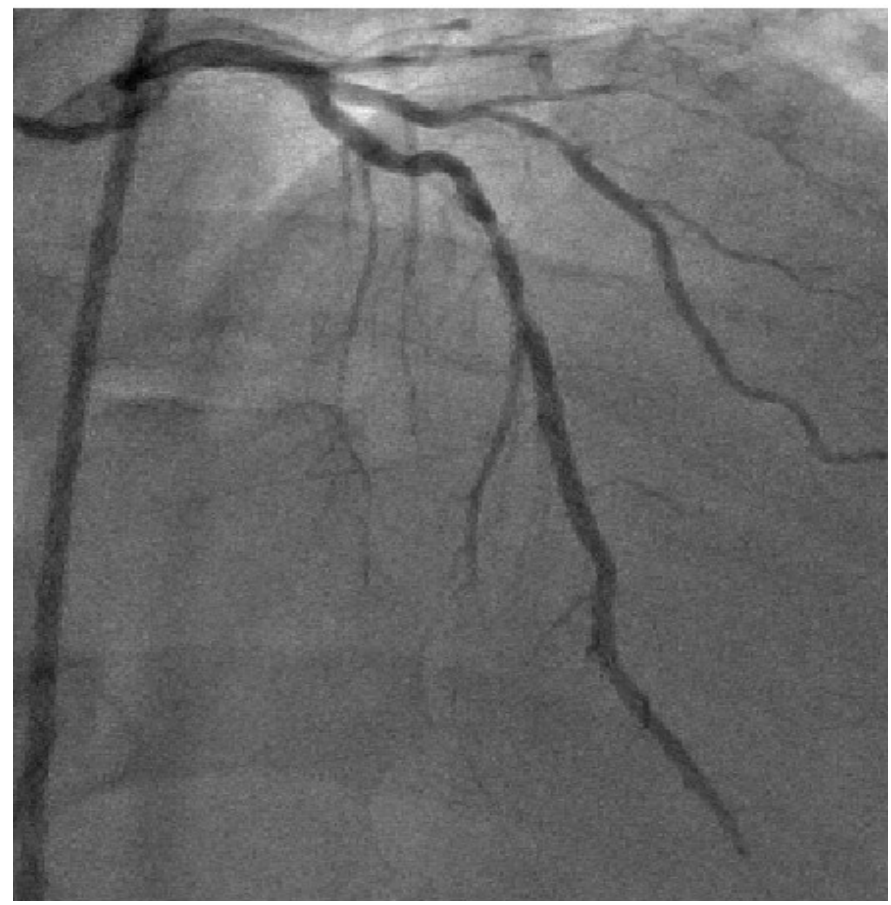

Figure 8. Coronary angiography showing diffuse atherosclerotic LAD with mid LAD lesion.

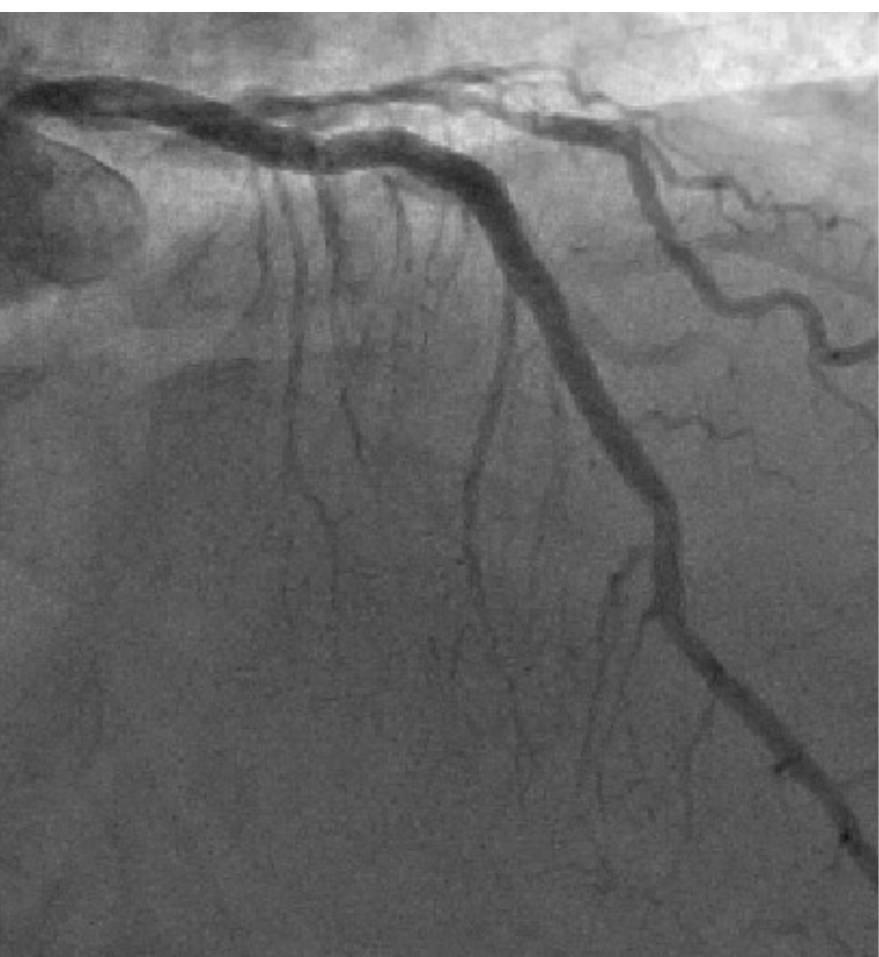

Figure 9. Coronary angiography showing the final results after stenting of the LAD. 


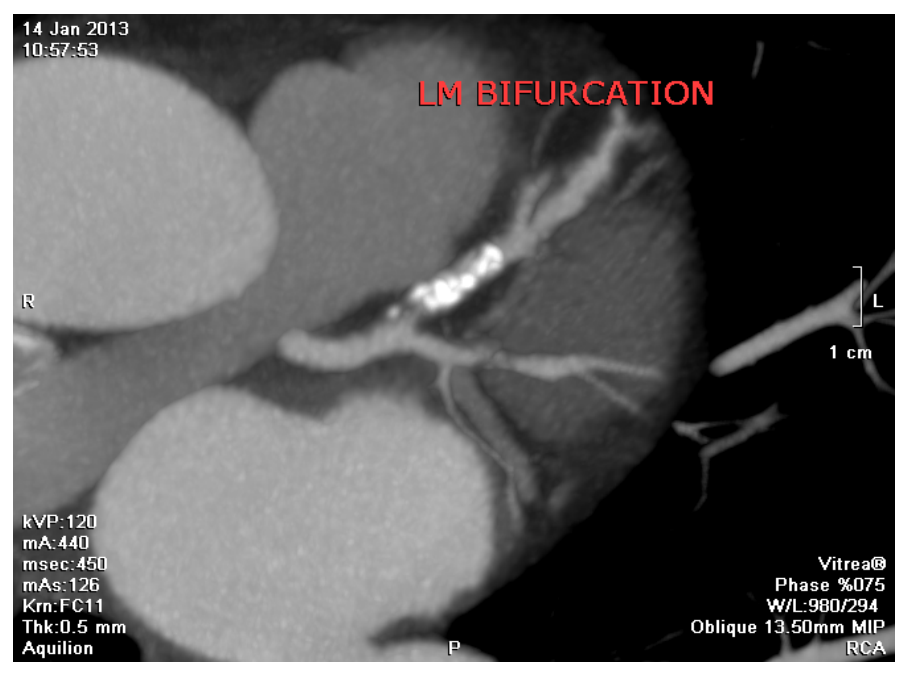

Figure 10. MPR image of LMCA bifurcation; showing highly calcific LAD plaque. There is another soft plaque in the OM branch.

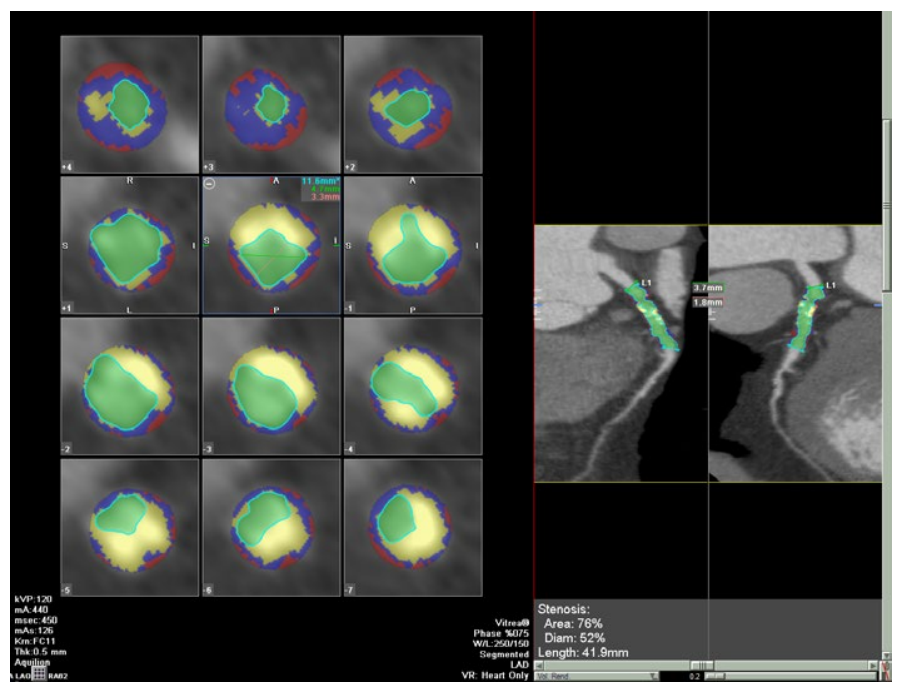

Figure 11. MPR imaging of the LAD plaque. The cross section showed dense calcium deposition (yellow color) in the vessel wall.

\section{Results}

\section{Base line characteristics}

In this study we enrolled 45 patients with male gender predominance; $82.2 \%$ of them were males and $17.8 \%$ were females. The mean age was $59.73 \pm 9.93$ years. Risk factors of coronary artery disease were frequently observed among enrolled subjects. Of the study group, 53.3\% were diabetics while hypertension was encountered in about $80 \%$ of patients. $62.2 \%$ of patients were suffering from dyslipedemia and $46.7 \%$ of the study group were smokers. Only $11.1 \%$ of the patients had positive family history of premature coronary artery disease.

\section{Procedural characteristics}

Sixty lesions were attempted; 3 (5\%) were LMCA, 29 (48.3\%) were LAD, 11 (18.3\%) were LCX and 17 (28.3\%) were RCA lesions.

Complex PCI as specifically defined above was reported in 43 interventions (72\%). Buddy wire was used in 1 intervention $(2.3 \%)$, kissing balloon in 3 (7\%), balloon pre-dilatation in 20 (45.5\%), balloon post-dilatation in $30(69.8 \%)$, anchor technique in $1(2.3 \%)$ and rotablator in $2(4.7 \%)$. Procedure related complications were observed in 7 interventions (11.7\%) with dissection in 4 interventions $(6.7 \%)$; three of them were of calcific plaque type and the remaining one was mixed plaque. However, all of them required repeated balloon post dilatation using high pressure balloon. No reflow phenomenon was reported in 1 intervention (1.7\%) after development of flow limiting dissection and plaque shift was observed in 3 interventions (5\%), all of them were of soft plaque type. These procedural complications were successfully managed without any adverse consequences for the patients.

\section{CCTA derived parameters indicate complexity of PCI}

Median Agatston score was significantly higher in lesions with complex as compared to those with non-complex interventions with a mean of $266.51 \pm 155.93$ vs. $168.76 \pm 70.53$ with the $\mathrm{P}$ value $=0.034$

Regarding plaque density, Hounsfield Units were higher in lesions with complex as compared to non-complex PCI (371.85 (48.0 to 1159.0) vs. 232.29 (52.0 to 1307.0) Hounsfield Units; $P=.033$ ).

Median local plaque volume was higher in lesions with complex PCI compared to those with non-complex interventions with a mean of 475.07 (92.0 to 1229.0$) \mathrm{mm}^{3}$ vs. 270.24 (69.0 to 824.0$) \mathrm{mm}^{3}$.

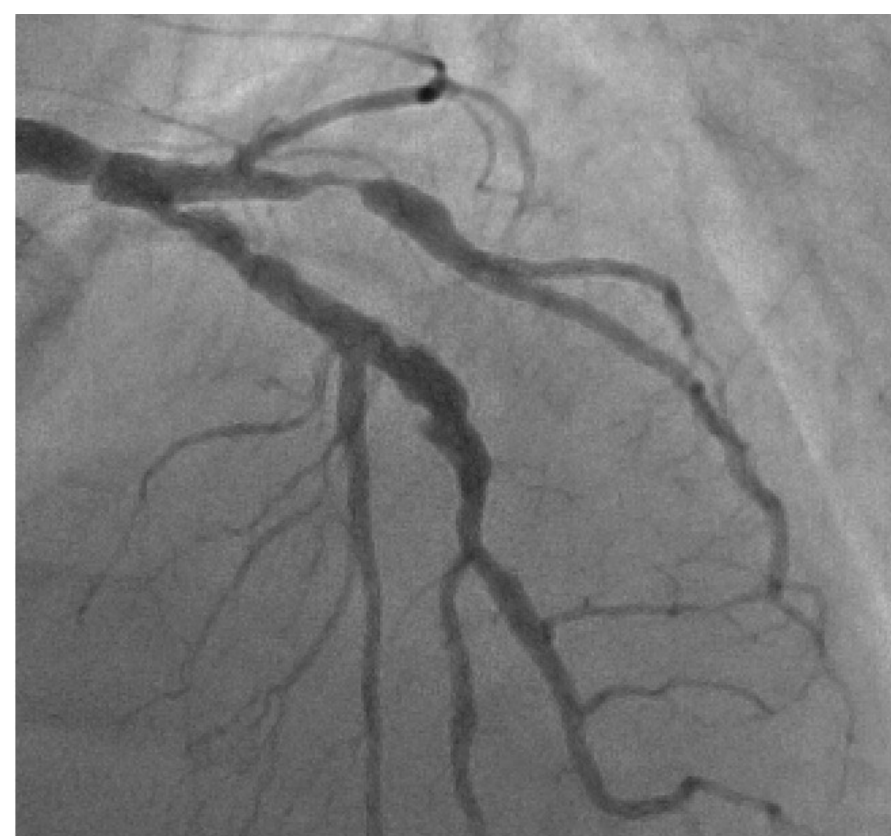

Figure 12. LAO view showing very tight ostial LAD lesion. The early OM showed proximal tight lesion.
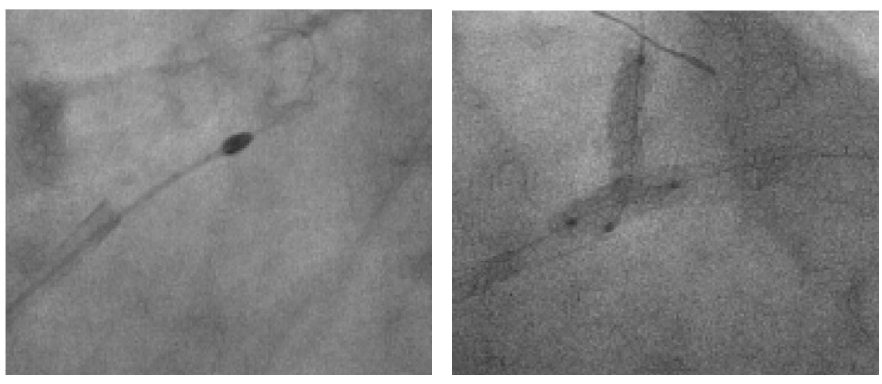

Figure 13. Rota ablator and kissing balloon techniques. 


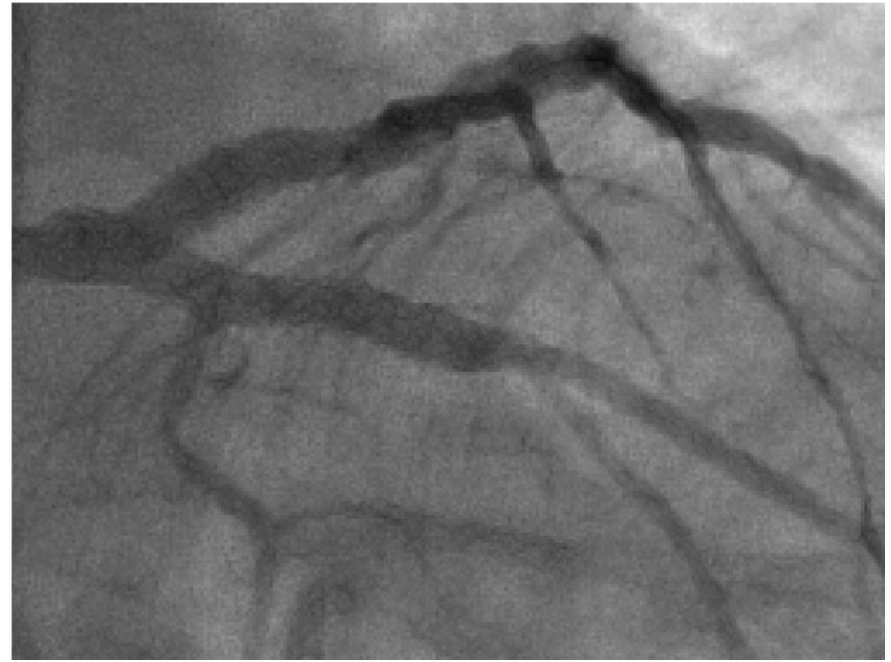

Figure 14. RAO view showing well deployed LM-CX and LAD stents.

The plaque area was found statistically significantly higher in complex PCI procedures over the non-complex ones with the mean plaque area in the complex procedures was $19.40 \pm 14.61 \mathrm{~mm}^{2}$ and in the non-complex ones, it was $11.24 \pm 7.94 \mathrm{~mm}^{2}$ with the $\mathrm{P}$ value $=0.011$.

Complex PCI had a higher rate of calcified plaques $(27.9 \%$ vs. 5.9\%; $P=.046)$.

On the other hand; side branch involvement, lesion morphology, degree of stenosis and plaque length did not show statistically significant correlation with PCI complexity.

In the next section we will demonstrate some examples of complex procedures enrolled in our study.

\section{Case number 1 (Patient number 1)}

A male patient, 62 years old with history of diabetes mellitus, hypertension and dyslipedemia presented with atypical anginal pains.

His coronary CT angiography revealed a long proximal to mid calcific plaque in the LAD. The plaque characteristics were as following; length was $56 \mathrm{~mm}$, volume was $634 \mathrm{~mm}^{3}$, area was $19.3 \mathrm{~mm}^{2}$, density was $465 \mathrm{HU}$, local plaque calcium score was 587 and degree of stenosis was $63 \%$.

Then, patient underwent PCI LAD after one week. It was a very complex procedure which necissated the use of IVUS which confirmed the heavy calcium burden. The operator used Rota ablator, balloon pre and post dilatation several times in order to get a satisfactory final result. He used 2 successive drug eluting stents without leaving a gap in between; their dimensions were $2.75 \times 38 \mathrm{~mm}$ and $3 \times 23 \mathrm{~mm}$.

\section{Case number 2 (Patient number 2)}

A male patient 60 years old, hypertensive, dyslipedemic and smoker presented with stable angina. The coronary CT angiography revealed ostial and Para ostial LAD highly calcific plaque. The ostium of circumflex branch $(\mathrm{Cx})$ was spared with a very proximal highly obstructive soft plaque in an early obtuse marginal branch (OM). The LAD plaque characteristics were as following; length was $36.4 \mathrm{~mm}$, volume was $757 \mathrm{~mm}^{3}$, area was $23.8 \mathrm{~mm}^{2}$, density was $156 \mathrm{HU}$ and maximum site of stenosis was $81 \%$. The local plaque calcium burden was 479 . The OM plaque was soft plaque (calcium score $=3$ ). Its length was $33 \mathrm{~mm}$, volume was $349 \mathrm{~mm}^{3}$, area was $17.2 \mathrm{~mm}^{2}$, density was 76 $\mathrm{HU}$ and maximum site of stenosis was $76 \%$.

Then, patient underwent a very complex PCI procedure using rotational atherectomy of the proximal LAD plaque and several balloon pre dilatation trials. Then using culotte technique with $4 \mathrm{x}$ $32 \mathrm{~mm}$ stent used to cover the OM plaque landed proximally at the distal LMCA. Another stent $3 \times 28 \mathrm{~mm}$ was deployed at the LAD ostial plaque. Kissing balloon technique was used for proximal optimization of the newly made carina with very good final results.

\section{Conclusion}

The proposed pre-procedural CCTA parameters indicate complexity of PCI. Thus, we suggest that in patients with suspected complex coronary anatomy, prior CCTA adds important complementary information to coronary angiography for planning subsequent PCI strategy.

\section{References}

1. Serruys PW, Morice MC, Kappetein AP, Colombo A, Holmes DR, et al. (2009) Percutaneous coronary intervention versus coronary-artery bypass grafting for severe coronary artery disease. $N$ Engl J Med 360: 961-972. [Crossref]

2. Valgimigli M, Serruys PW, Tsuchida K, Vaina S, Morel MA, et al. (2007) Cyphering the complexity of coronary artery disease using the syntax score to predict clinical outcome in patients with three-vessel lumen obstruction undergoing percutaneous coronary intervention. Am J Cardiol 99: 1072-1081. [Crossref]

3. Capodanno D, Capranzano P, Di Salvo ME, Caggegi A, Tomasello D, et al. (2009) Usefulness of SYNTAX score to select patients with left main coronary artery disease to be treated with coronary artery bypass graft. JACC Cardiovasc Interv 2: 731-738. [Crossref]

4. von Ballmoos MW, Haring B, Juillerat P, Alkadhi H (2011) Meta-analysis: diagnostic performance of low-radiation-dose coronary computed tomography angiography. Ann Intern Med 154: 413-420. [Crossref]

5. Magro M, Schultz C, Simsek C, Garcia-Garcia HM, Regar E, et al. (2010) Computed tomography as a tool for percutaneous coronary intervention of chronic total occlusions. Euro Intervention 6 Suppl G: G123-131. [Crossref]

6. Yokoyama N, Yamamoto Y, Suzuki S, Suzuki M, Konno K, et al. (2006) Impact of 16-slice computed tomography in percutaneous coronary intervention of chronic total occlusions. Catheter Cardiovasc Interv 68: 1-7. [Crossref]

7. Hecht HS (2008) Applications of multislice coronary computed tomographic angiography to percutaneous coronary intervention: how did we ever do without it? Catheter Cardiovasc Interv 71: 490-503. [Crossref]

8. Mollet NR, Hoye A, Lemos PA, Cademartiri F, Sianos G, et al. (2005) Value of preprocedure multislice computed tomographic coronary angiography to predict the outcome of percutaneous recanalization of chronic total occlusions. Am J Cardiol 95: 240-243. [Crossref]

9. Li P, Gai LY, Yang X, Sun ZJ, Jin QH (2010) Computed tomography angiographyguided percutaneous coronary intervention in chronic total occlusion. J Zhejiang Univ Sci B 11: 568-574. [Crossref]

10. García-García HM, van Mieghem CA, Gonzalo N, Meijboom WB, Weustink AC, et al (2009) Computed tomography in total coronary occlusions (CTTO registry): radiation exposure and predictors of successful percutaneous intervention. Euro Intervention 4 : 607-616. [Crossref]

11. Soon KH, Cox N, Wong A, Chaitowitz I, Macgregor L, et al. (2007) CT coronary angiography predicts the outcome of percutaneous coronary intervention of chronic total occlusion. J Interv Cardiol 20: 359-366. [Crossref]

12. Ehara M, Terashima M, Kawai M, Matsushita S, Tsuchikane E, et al. (2009) Impact of multislice computed tomography to estimate difficulty in wire crossing in percutaneous coronary intervention for chronic total occlusion. J Invasive Cardiol 21: 575-582. [Crossref]

13. Hsu JT, Kyo E, Chu CM, Tsuji T, Watanabe S (2011) Impact of calcification length ratio on the intervention for chronic total occlusions. Int J Cardiol 150: 135-141. [Crossref]

14. Cho JR, Kim YJ, Ahn CM, Moon JY, Kim JS, et al. (2010) Quantification of regiona calcium burden in chronic total occlusion by 64 -slice multi-detector computed 
Ayad SW (2015) Role of plaque characterization by 64-slice multi detector computed tomography in prediction of complexity of percutaneous coronary interventions

tomography and procedural outcomes of percutaneous coronary intervention. Int $J$ Cardiol 145: 9-14. [Crossref]

15. Zhang F, Dong L, Ge J (2009) Simple versus complex stenting strategy for coronary artery bifurcation lesions in the drug-eluting stent era: a meta-analysis of randomised trials. Heart 95: 1676-1681. [Crossref]

16. Sharma SK, Choudhury A, Lee J, Kim MC, Fisher E, et al. (2004) Simultaneous kissing stents (SKS) technique for treating bifurcation lesions in medium-to-large size coronary arteries. Am J Cardiol 94: 913-917. [Crossref]

Copyright: $@ 2015$ Ayad SW. This is an open-access article distributed under the terms of the Creative Commons Attribution License, which permits unrestricted use, distribution, and reproduction in any medium, provided the original author and source are credited. 